\title{
Optimal Distributed Detection for Complex Field Network Coding Assisted Wireless Sensor Networks with Nonorthogonal Communications
}

\author{
Yulun Cheng, Longxiang Yang, and Hongbo Zhu \\ Jiangsu Key Lab of Wireless Communications, Nanjing University of Posts and Telecommunications, Nanjing 210003, China \\ Correspondence should be addressed to Yulun Cheng; chengyuluen@163.com
}

Received 25 May 2016; Revised 13 October 2016; Accepted 26 October 2016

Academic Editor: Paolo Bellavista

Copyright (C) 2016 Yulun Cheng et al. This is an open access article distributed under the Creative Commons Attribution License, which permits unrestricted use, distribution, and reproduction in any medium, provided the original work is properly cited.

Complex field network coding (CFNC) enables signal transmission and information fusion to be operated over nonorthogonal channels, which essentially improves both the bandwidth efficiency and transmission reliability of the wireless sensor networks (WSNs). This paper investigates the power allocation for the CFNC assisted WSNs. By improving the accuracy of the approximation for $Q$ function, the closed-form expression of the pairwise error probability bound for the considered network is derived, so as to improve the optimization efficiency of the power allocation. On the basis of the expression, the relay power coefficient is optimized under the fixed power constraint. Besides, the impacts of the topology parameters on the power allocation are also analyzed accordingly. Simulations demonstrate that the proposed power allocation outperforms the benchmark in terms of the detection performance. The obtained expression of the power coefficient and insights can provide useful suggestions for the power allocation design of the CFNC assisted WSNs.

\section{Introduction}

Due to the economical deployment and wide range of applications [1], wireless sensor networks (WSNs) have drawn an upsurge of research interests in recent years. Generally, a WSN consists of low powered sensor nodes, which only own constrained processing and communication capabilities. This fact puts limits on the WSNs in both the bandwidth efficiency and transmission reliability in wireless fading channels [2].

Distributed detection (DD) [3] is an efficient solution for the limitation of WSNs. In the DD, each sensor node transmits no raw data but the decision extracted from it, which help to reduce bandwidth expense. Besides, DD is also able to combat the adverse effects of wireless fading channels, because the same raw data are observed by the decisions of multiple sensor nodes and transmitted to the fusion center through multiple independent channels. For the above merits, DD has been extensively studied by the literatures. For example, towards the scenarios that the decisions of the sensors are conditionally independent $[4,5]$ and correlated $[6,7]$, the optimal DD and decision fusion have been analyzed, respectively. However, in these works, the communication is assumed to be error-free. References [8,9] investigate the effects of the noise and wireless fading in the decision fusion, in which the channel state information (CSI) is assumed to be fully known at the receivers. Later, the case is also studied in [10] when only partial CSI is available.

In these previous studies, to avoid multiaccess interference (MAI), all the sensor nodes transmit in the manner of orthogonal communications, where only one sensor node is permitted to transmit at a certain time. This manner leads to the inefficient utilization of the bandwidth and constrains the throughput of the network, especially for the large WSNs. Therefore, nonorthogonal communications- (NOC-) enabled DD [11-14] appears. With NOC, all the sensor nodes transmit simultaneously over the multiaccess channel, so the bandwidth efficiency is greatly improved.

Interfered by the MAI, the transmission reliability is a key issue for the NOC-enabled DD. Recent work [15] employs complex field network coding (CFNC) [16] as a solution of NOC, in which the cooperative relay $[17,18]$ is also integrated. On the one hand, by using the cooperative relay, the diversity 
gain can be obtained to improve the transmission reliability. On the other hand, through power allocation, the influence of MAI is also mitigated. Hence, by combing CFNC and cooperative relay, [15] provides an efficient solution in both bandwidth and transmission reliability for WSNs.

In the power allocation of [15], the critical step is to obtain a closed-form expression of the detection performance. However, to yield a traceable expression of the pairwise error probability (PEP) bound, the Chernoff bound [19] is utilized to approximate $Q$ function in the computation [15], which is not very tight [20] and may cause degradation to the optimized result.

This paper focuses on the optimization of the power allocation for the CFNC assisted WSNs, and the major contributions are summarized as follows:

(i) Contrary to the former work [15], we employ the result of [20] to improve the accuracy of approximation for $Q$ function and derive the closed-form expression of the PEP bound for the considered network.

(ii) We utilize the obtained expression to optimize the relay power by considering fixed power budget. Moreover, the impacts of the topology parameters are also analyzed accordingly, through which the relationship between the power allocation and node location is revealed.

(iii) We examine the performance of the obtained result by comparing it with the one of [15].

The rest of this paper is organized as follows. Section 2 presents the system model of CFNC assisted DD. In Section 3, the PEP analysis and power optimization are presented. Simulation results are provided in Section 4. Finally, Section 5 concludes the paper.

\section{System Model of CFNC Assisted DD}

We consider the CFNC assisted DD in a parallel WSN, which is utilized to monitor the region of interest (ROI) through binary hypotheses $H_{1}$ and $H_{0}$. The network is consisted of $N$ sensor nodes, one fusion center (FC), and one relay node $R$. As is shown in Figure 1, each sensor node $S_{k}$ firstly acquires raw measurement from the ROI to generate its decision $u_{k}$ ( 1 or 0$)$, which is then modulated to produce its signal $x_{k}(-1$ or 1$)$. Here, the sensing accuracy of $S_{k}$ is described by the probability of detection $P_{D k}$ and probability of false alarm $P_{F k}$, which are expressed as

$$
\begin{aligned}
& P_{D k}=\operatorname{Pr}\left(x_{k}=1 \mid H_{1}\right), \\
& P_{F k}=\operatorname{Pr}\left(x_{k}=1 \mid H_{0}\right) .
\end{aligned}
$$

After that, all $N$ signals are transmitted to $\mathrm{FC}$, which are distorted by the fading and noise. Finally, FC combines all the received signals and casts a final decision.

With orthogonal communications, it costs $N$ time slots (TS) to transmit all $N$ signals. In contrast, with CFNC assisted NOC, only two TSs are needed. According to the scheme,

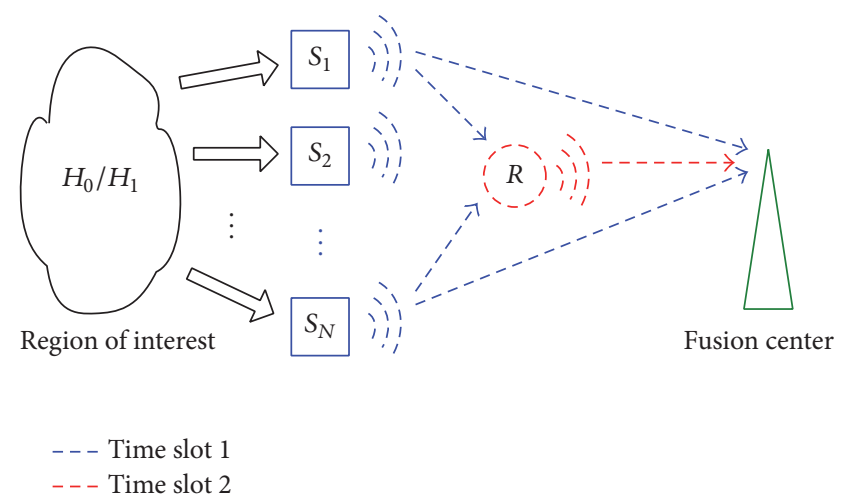

FIGURE 1: System model of CFNC assisted DD.

each $S_{k}$ owns a unique signature $\theta_{k}$. In TS 1 , each $S_{k}$ produces its CFNC signal by multiplying $\theta_{k}$ and $x_{k}$ and then transmits $\theta_{k} x_{k}$ simultaneously. Hence, N CFNC signals overlap at both $R$ and FC, which can be expressed as

$$
\begin{aligned}
& y_{s r}=\sum_{k=1}^{N} \sqrt{r_{k}} h_{k r} \theta_{k} x_{k}+z_{r}, \\
& y_{s d}=\sum_{k=1}^{N} \sqrt{g_{k}} h_{k d} \theta_{k} x_{k}+z_{d},
\end{aligned}
$$

where $y_{s r}$ and $y_{s d}$ are the received signals at $R$ and FC, respectively. $r_{k}$ and $g_{k}$ denote the path loss coefficient of $S_{k}$-to- $R$ and $S_{k}$-to-FC links, respectively, while $h_{k r}$ and $h_{k d}$ capture the corresponding fading coefficients. Throughout the manuscript, we assume that the channel state information (CSI) is available at all the receivers. $z_{r}$ and $z_{d}$ denote the background noise at $R$ and FC, respectively, which are modeled as additive white Gaussian noise (AWGN) with zero mean and variance of $N_{0}$. After that, $R$ decodes the received signals by maximum likelihood (ML) detection, which is

$$
\left(\widehat{x}_{1}, \ldots, \widehat{x}_{N}\right)=\underset{x_{1}, \ldots, x_{N}}{\arg \min }\left\|y_{s r}-\sum_{k=1}^{N} \sqrt{r_{k}} h_{k r} \theta_{k} x_{k}\right\|^{2} .
$$

Then, $R$ encodes the obtained signals as

$$
x_{r}=\sum_{k=1}^{N} \theta_{k} \widehat{x}_{k}
$$

and transmits $x_{r}$ to FC in TS 2. So the received signal at FC can be written as

$$
y_{r d}=\sqrt{a r_{r}} h_{r d} x_{r}+z_{d}
$$

where coefficient $a$ denotes the power allocated to $R$.

Finally, FC combines all the received signals in TS 1 and 2. With the optimal fusion rule in Bayesian sense, the corresponding likelihood ratio is computed as

$$
\begin{aligned}
& \widehat{\Lambda}\left(y_{s d}, y_{r d}\right) \\
& =\frac{\sum_{\mathbf{x}} f\left(y_{s d} \mid H_{1}, \mathbf{X}\right)\left(\sum_{\widehat{\mathbf{x}}} f\left(y_{r d} \mid H_{1}, \widehat{\mathbf{x}}\right)\right) P(\widehat{\mathbf{X}} \mid \mathbf{x}) P\left(\mathbf{X} \mid H_{1}\right)}{\sum_{\mathbf{X}} f\left(y_{s d} \mid H_{0}, \mathbf{X}\right)\left(\sum_{\widehat{\mathbf{x}}} f\left(y_{r d} \mid H_{0}, \widehat{\mathbf{X}}\right)\right) P(\widehat{\mathbf{X}} \mid \mathbf{X}) P\left(\mathbf{X} \mid H_{0}\right)},
\end{aligned}
$$


where $\mathbf{X}=\left[x_{1}, \ldots, x_{N}\right]^{T}, \widehat{\mathbf{X}}=\left[\widehat{x}_{1}, \ldots, \widehat{x}_{N}\right]^{T}$. To simplify the analysis, we follow the assumption utilized in [15]; that is, $\widehat{\mathbf{X}}=$ $\mathbf{X}$. Thus, (6) can be simplified as

$$
\begin{aligned}
& \Lambda\left(y_{s d}, y_{r d}\right) \\
& \quad=\frac{\sum_{\mathbf{X}} f\left(y_{s d} \mid H_{1}, \mathbf{X}\right) f\left(y_{r d} \mid H_{1}, \mathbf{X}\right) P\left(\mathbf{X} \mid H_{1}\right)}{\sum_{\mathbf{X}} f\left(y_{s d} \mid H_{0}, \mathbf{X}\right) f\left(y_{r d} \mid H_{0}, \mathbf{X}\right) P\left(\mathbf{X} \mid H_{0}\right)} .
\end{aligned}
$$

When $\mathbf{X}$ is given, the conditional probability of $y_{s d}\left(y_{r d}\right)$ is independent of the hypothesis, so (7) can be simplified as

$$
\Lambda\left(y_{s d}, y_{r d}\right)=\frac{\sum_{\mathbf{X}} f\left(y_{s d} \mid \mathbf{X}\right) f\left(y_{r d} \mid \mathbf{X}\right) P\left(\mathbf{X} \mid H_{1}\right)}{\sum_{\mathbf{X}} f\left(y_{s d} \mid \mathbf{X}\right) f\left(y_{r d} \mid \mathbf{X}\right) P\left(\mathbf{X} \mid H_{0}\right)} .
$$

According to the optimal likelihood ratio based fusion rule [9], the condition distributions and probabilities in (8) can be given as

$$
\begin{array}{r}
f\left(y_{s d} \mid \mathbf{X}\right) \\
=\frac{1}{2 \pi \sigma^{2}} \exp \left(-\frac{\left|y_{s d}-\sum_{k=1}^{N} \sqrt{g_{k}} h_{k d} \theta_{k} x_{k}\right|^{2}}{2 \sigma^{2}}\right), \\
f\left(y_{r d} \mid \mathbf{X}\right)=\frac{1}{2 \pi \sigma^{2}} \exp \left(-\frac{\left|y_{r d}-\sqrt{a r_{r}} h_{r d} x_{r}\right|^{2}}{2 \sigma^{2}}\right), \\
P\left(\mathbf{X} \mid H_{1}\right)=\prod_{k=1}^{N} P_{D k}^{u_{k}}\left(1-P_{D k}\right)^{1-u_{k}}, \\
P\left(\mathbf{X} \mid H_{0}\right)=\prod_{k=1}^{N} P_{F k}^{u_{k}}\left(1-P_{F k}\right)^{1-u_{k}} .
\end{array}
$$

And then, FC generates the final decision as

$$
\begin{array}{ll}
H_{1} \text { is ture, } & \text { if } \Lambda\left(y_{s d}, y_{r d}\right) \geq t, \\
H_{0} \text { is ture, } & \text { if } \Lambda\left(y_{s d}, y_{r d}\right)<t,
\end{array}
$$

where $t$ is the detection threshold. By means of minimizing the error detection probability, the optimal value of $t$ can be obtained as

$$
t=\frac{P\left(H_{0}\right)}{P\left(H_{1}\right)}
$$

where $P\left(H_{1}\right)$ and $P\left(H_{0}\right)$ are a priori probabilities of $H_{0}$ and $H_{1}$, respectively. Thus, the detection metrics of the scheme can be expressed as

$$
\begin{aligned}
& P_{F}=P\left(\Lambda\left(y_{s d}, y_{r d}\right) \geq t \mid H_{0}\right), \\
& P_{D}=P\left(\Lambda\left(y_{s d}, y_{r d}\right) \geq t \mid H_{1}\right),
\end{aligned}
$$

where $P_{F}$ and $P_{D}$ are the false alarm probability and detection probability, respectively. Thus, the average error probability can be expressed as

$$
P_{e}=P_{F} P\left(H_{0}\right)+\left(1-P_{D}\right) P\left(H_{1}\right) .
$$

\section{PEP Analysis and Power Allocation}

In this section, we determine the sensor signature $\boldsymbol{\theta}=$ $\left[\theta_{1}, \ldots, \theta_{N}\right]^{T}$ and the relay power coefficient $a$ by optimizing the ML bound on the PEP of the considered network. Since each sensor detection is binary, $\mathbf{X}$ has $2^{N}$ possibilities, and we denote $\mathbf{X}_{i}$ as $i$ th possible detection vector, while its corresponding CFNC symbol can be expressed as $C_{i}=\boldsymbol{\theta}^{T} \mathbf{X}_{i}$. Thus, the PEP at FC can be written as

$$
\begin{aligned}
& \mathrm{PEP}_{F} \triangleq P\left(C_{i} \longrightarrow C_{i} \text { at } R \mid C_{i}\right) \\
& \cdot P\left(C_{i} \longrightarrow C_{j} \text { at } \mathrm{FC} \mid C_{i} \longrightarrow C_{i} \text { at } R, C_{i}\right) \\
& +P\left(C_{i} \longrightarrow C_{j} \text { at } R \mid C_{i}\right) \\
& \cdot\left(1-P\left(C_{i} \longrightarrow C_{i} \text { at } \mathrm{FC} \mid C_{i} \longrightarrow C_{j} \text { at } R, C_{i}\right)\right),
\end{aligned}
$$

where $P\left(C_{i} \rightarrow C_{i}\right.$ at $\left.R \mid C_{i}\right)$ and $P\left(C_{i} \rightarrow C_{j}\right.$ at $\left.R \mid C_{i}\right)$ are the correctly decoding probability and PEP at $R$ when $C_{i}$ is given, respectively. $P\left(C_{i} \rightarrow C_{j}\right.$ at $\mathrm{FC} \mid C_{i} \rightarrow C_{i}$ at $\left.R, C_{i}\right)$ is the PEP at FC given that $C_{i}$ is sent and $R$ decodes correctly. $P\left(C_{i} \rightarrow C_{i}\right.$ at FC | $C_{i} \rightarrow C_{j}$ at $\left.R, C_{i}\right)$ is the correctly decoding probability at FC given that $C_{i}$ is sent and $R$ decodes incorrectly.

When the CSIs are known at $R$, its PEP can be written as

$$
\begin{aligned}
P & \left(C_{i} \longrightarrow C_{j} \text { at } R \mid C_{i}\right) \\
& =P\left(\left|\sum_{k=1}^{N} \sqrt{r_{k}} h_{k r} \theta_{k}\left(X_{i}^{(k)}-X_{j}^{(k)}\right)+z_{r}\right|^{2} \leq\left|z_{r}\right|^{2}\right) \\
& =P\left(z_{r}^{*}\left(\sum_{k=1}^{N} \sqrt{r_{k}} h_{k r} \theta_{k} d_{i j}^{(k)}\right)\right. \\
& \left.+z_{r}\left(\sum_{k=1}^{N} \sqrt{r_{k}} h_{k r} \theta_{k} d_{i j}^{(k)}\right)^{*} \geq\left|\sum_{k=1}^{N} \sqrt{r_{k}} h_{k r} \theta_{k} d_{i j}^{(k)}\right|^{2}\right),
\end{aligned}
$$

where “*” stands for complex conjugate; $X_{i}^{(k)}$ and $d_{i j}^{(k)}$ are $k$ th component of $\mathbf{X}_{i}$ and $\left(\mathbf{X}_{i}-\mathbf{X}_{j}\right)$, respectively. Thus, $z_{r}^{*}\left(\sum_{k=1}^{N}\right.$ $\left.\sqrt{r_{k}} h_{k r} \theta_{k} d_{i j}^{(k)}\right)+z_{r}\left(\sum_{k=1}^{N} \sqrt{r_{k}} h_{k r} \theta_{k} d_{i j}^{(k)}\right)^{*}$ follow the Gaussian distribution with zero mean and $4 \sigma^{2}\left|\sum_{k=1}^{N} \sqrt{r_{k}} h_{k r} \theta_{k} d_{i j}^{(k)}\right|^{2}$ variance, so (15) can be expressed as

$$
\begin{aligned}
P & \left(C_{i} \longrightarrow C_{j} \text { at } R \mid C_{i}\right) \\
& =Q\left(\frac{\left|\sum_{k=1}^{N} \sqrt{r_{k}} h_{k r} \theta_{k} d_{i j}^{(k)}\right|}{2 \sigma}\right) .
\end{aligned}
$$


Besides, the PEP at FC given that $R$ correctly decodes $C_{i}$ can be expressed as

$$
\begin{aligned}
P & \left(C_{i} \longrightarrow C_{j} \text { at } \mathrm{FC} \mid C_{i} \longrightarrow C_{i} \text { at } R, C_{i}\right) \\
& =P\left(\left|\sum_{k=1}^{N} \sqrt{g_{k}} h_{k d} \theta_{k}\left(X_{i}^{(k)}-X_{j}^{(k)}\right)+z_{d}\right|^{2}\right. \\
& \left.+\left|\sqrt{a r_{r}} h_{r d} \sum_{k=1}^{N} \theta_{k}\left(X_{i}^{(k)}-X_{j}^{(k)}\right)+z_{d}\right|^{2} \leq 2\left|z_{d}\right|^{2}\right) .
\end{aligned}
$$

By a similar analysis as in (15), (17) can be expressed as

$$
P\left(C_{i} \longrightarrow C_{j} \text { at } \mathrm{FC} \mid C_{i} \longrightarrow C_{i} \text { at } R, C_{i}\right)
$$

$$
=Q\left(\frac{\sqrt{\left|\sum_{k=1}^{N} \sqrt{g_{k}} h_{k d} \theta_{k} d_{i j}^{(k)}\right|^{2}+\left|\sqrt{a_{r}} h_{r d} \sum_{k=1}^{N} \theta_{k} d_{i j}^{(k)}\right|^{2}}}{2 \sigma}\right) .
$$

Also, the correct decoding probability at FC given, that $R$ decodes erroneously, can be obtained as

$$
\begin{aligned}
& P\left(C_{i} \longrightarrow C_{i} \text { at } \mathrm{FC} \mid C_{i} \longrightarrow C_{j} \text { at } R, C_{i}\right) \\
& =Q\left(\frac{\left|\sqrt{a r_{r}} h_{r d} \sum_{k=1}^{N} \theta_{k} d_{i j}^{(k)}\right|^{2}-\left|\sum_{k=1}^{N} \sqrt{g_{k}} h_{k d} \theta_{k} d_{i j}^{(k)}\right|^{2}}{2 \sigma \sqrt{\left|\sum_{k=1}^{N} \sqrt{g_{k}} h_{k d} \theta_{k} d_{i j}^{(k)}\right|^{2}+\left|\sqrt{a r_{r}} h_{r d} \sum_{k=1}^{N} \theta_{k} d_{i j}^{(k)}\right|^{2}}}\right) .
\end{aligned}
$$

By substituting (16), (18), and (19) into (14), the instantaneous $\mathrm{PEP}$ at FC can be obtained as

$$
\begin{aligned}
\operatorname{PEP}_{F}= & Q\left(\frac{\sqrt{\left|\sum_{k=1}^{N} \sqrt{g_{k}} h_{k d} \theta_{k} d_{i j}^{(k)}\right|^{2}+\left|\sqrt{a r_{r}} h_{r d} \sum_{k=1}^{N} \theta_{k} d_{i j}^{(k)}\right|^{2}}}{2 \sigma}\right) \times\left(1-Q\left(\frac{\left|\sum_{k=1}^{N} \sqrt{r_{k}} h_{k r} \theta_{k} d_{i j}^{(k)}\right|}{2 \sigma}\right)\right) \\
& +Q\left(\frac{\left|\sum_{k=1}^{N} \sqrt{r_{k}} h_{k r} \theta_{k} d_{i j}^{(k)}\right|}{2 \sigma}\right) \times\left(1-Q\left(\frac{\left|\sqrt{a r_{r}} h_{r d} \sum_{k=1}^{N} \theta_{k} d_{i j}^{(k)}\right|^{2}-\left|\sum_{k=1}^{N} \sqrt{g_{k}} h_{k d} \theta_{k} d_{i j}^{(k)}\right|^{2}}{2 \sigma \sqrt{\left|\sum_{k=1}^{N} \sqrt{g_{k}} h_{k d} \theta_{k} d_{i j}^{(k)}\right|^{2}+\left|\sqrt{a r_{r}} h_{r d} \sum_{k=1}^{N} \theta_{k} d_{i j}^{(k)}\right|^{2}}}\right)\right) .
\end{aligned}
$$

To obtain the traceable relationship between $\mathrm{PEP}_{F}$ and the power coefficient $a$, (20) is bounded as

$$
\begin{aligned}
& \operatorname{PEP}_{F} \\
& \leq Q\left(\frac{\sqrt{\left|\sum_{k=1}^{N} \sqrt{g_{k}} h_{k d} \theta_{k} d_{i j}^{(k)}\right|^{2}+\left|\sqrt{a r_{r}} h_{r d} \sum_{k=1}^{N} \theta_{k} d_{i j}^{(k)}\right|^{2}}}{2 \sigma}\right) \\
& \quad+Q\left(\frac{\left|\sum_{k=1}^{N} \sqrt{r_{k}} h_{k r} \theta_{k} d_{i j}^{(k)}\right|}{2 \sigma}\right) .
\end{aligned}
$$

A closed-form expression of $Q(x)$ is required to further simplify (21). Different from the previous work [15] in which the Chernoff bound [19] is utilized, we employ (1), (2) and (14) in [20] to approximate $Q(x)$ as

$$
Q(x) \approx \frac{\left((1 / 6) \exp \left(-x^{2} / 2\right)+(1 / 2) \exp \left(-2 x^{2} / 3\right)\right)}{2} .
$$

We utilize (22) on the purpose that the accuracy of the approximation for the derived closed-form expression can be improved, which is vital for the final optimization of $a$. Here, the accuracy comparison of the approximations are depicted in Figure 2, where $Q(x)$ is proposed as the benchmark. It shows that the gap between (22) and $Q(x)$ is narrower, which indicates that (22) is superior to the Chernoff bound in the accuracy of approximation.

Therefore, by utilizing (22), (21) can be simplified as

$$
\begin{aligned}
\operatorname{PEP}_{F} \leq & \frac{\left(\exp \left(-\left(\left|\sum_{k=1}^{N} \sqrt{g_{k}} h_{k d} \theta_{k} d_{i j}^{(k)}\right|^{2}+\left|\sqrt{a r_{r}} h_{r d} \sum_{k=1}^{N} \theta_{k} d_{i j}^{(k)}\right|^{2}\right) / 8 \sigma^{2}\right)+\exp \left(-\left|\sum_{k=1}^{N} \sqrt{r_{k}} h_{k r} \theta_{k} d_{i j}^{(k)}\right|^{2} / 8 \sigma^{2}\right)\right)}{12} \\
& +\frac{\left(\exp \left(-\left|\sum_{k=1}^{N} \sqrt{r_{k}} h_{k r} \theta_{k} d_{i j}^{(k)}\right|^{2} / 6 \sigma^{2}\right)+\exp \left(-\left(\left|\sum_{k=1}^{N} \sqrt{g_{k}} h_{k d} \theta_{k} d_{i j}^{(k)}\right|^{2}+\left|\sqrt{a r_{r}} h_{r d} \sum_{k=1}^{N} \theta_{k} d_{i j}^{(k)}\right|^{2}\right) / 6 \sigma^{2}\right)\right)}{4} .
\end{aligned}
$$




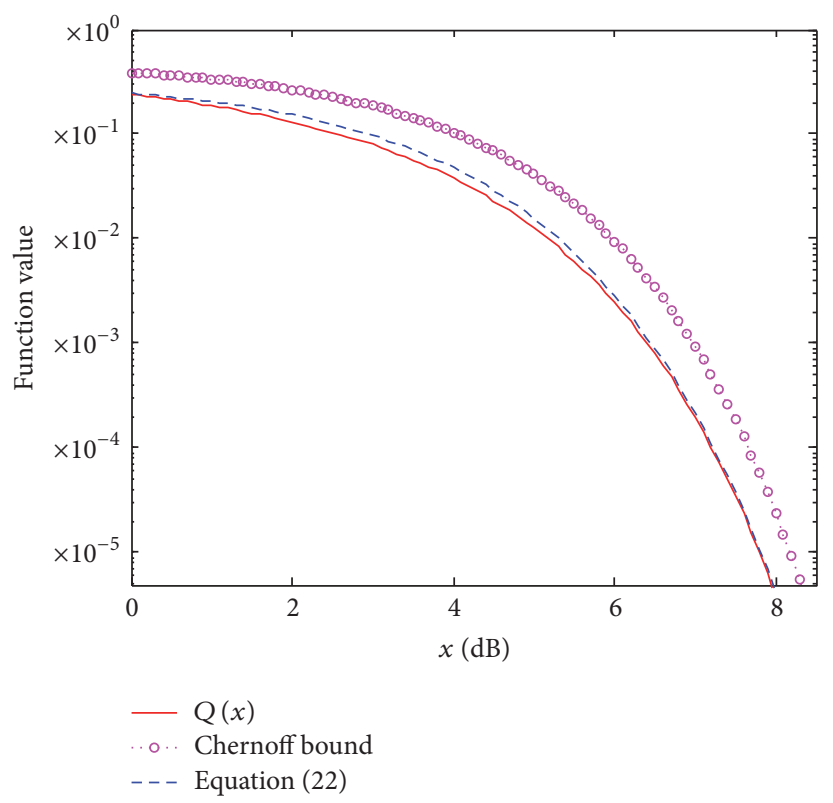

FIGURE 2: Accuracy comparison of approximation for $Q$ function.

We denote $w_{1}=\left|\sum_{k=1}^{N} \sqrt{g_{k}} h_{k d} \theta_{k} d_{i j}^{(k)}\right|^{2}, w_{2}=\mid \sqrt{a r_{r}} h_{r d}$ $\left.\sum_{k=1}^{N} \theta_{k} d_{i j}^{(k)}\right|^{2}, w_{3}=\left|\sum_{k=1}^{N} \sqrt{r_{k}} h_{k r} \theta_{k} d_{i j}^{(k)}\right|^{2}$. Since $h_{k d}, h_{r d}$, and $h_{k r}$ follow complex Gaussian distribution with zero mean and unit variance, $w_{1}, w_{2}$, and $w_{3}$ are exponent variables with a mean of $\lambda_{1}=\sum_{k=1}^{N} g_{k}\left|\theta_{k}\right|^{2}\left|d_{i j}^{(k)}\right|^{2}, \lambda_{2}=a r_{r}\left|\sum_{k=1}^{N} \theta_{k} d_{i j}^{(k)}\right|^{2}$, $\lambda_{3}=\sum_{k=1}^{N} r_{k}\left|\theta_{k}\right|^{2}\left|d_{i j}^{(k)}\right|^{2}$, respectively. Thus, the average PEP at FC can be bounded by averaging (23) over fading coefficients as

$$
\begin{aligned}
\overline{\mathrm{PEP}_{F}} & \leq \int_{0}^{\infty} \int_{0}^{\infty} \int_{0}^{\infty}\left(\frac{\left(\exp \left(-\left(w_{1}+w_{2}\right) / 8 \sigma^{2}\right)+\exp \left(-w_{3} / 8 \sigma^{2}\right)\right)}{12}+\frac{\left(\exp \left(-\left(w_{1}+w_{2}\right) / 6 \sigma^{2}\right)+\exp \left(-w_{3} / 6 \sigma^{2}\right)\right)}{4}\right) \\
& \times \prod_{i=1}^{3} \lambda_{i} \times \exp \left(-\sum_{i=1}^{3} \lambda_{i} w_{i}\right) d w_{1} d w_{2} d w_{3} \leq \frac{\left(\prod_{i=1}^{2}\left(1 /\left(1+\lambda_{i} / 8 \sigma^{2}\right)\right)+1 /\left(1+\lambda_{3} / 8 \sigma^{2}\right)\right)}{12} \\
& +\frac{\left(\prod_{i=1}^{2}\left(1 /\left(1+\lambda_{i} / 6 \sigma^{2}\right)\right)+1 /\left(1+\lambda_{3} / 6 \sigma^{2}\right)\right)}{4} .
\end{aligned}
$$

To make a fair comparison with [15], we similarly express the sensor signature in the polar form as $\theta_{i}=\sqrt{P_{i}} e^{j \varphi_{i}}$, where $P_{i}$ and $\varphi_{i}$ denote the transmit power and phase of $i$ th sensor signature, respectively. $\varphi_{i}$ is also determined by the heuristic method in [15]. Thus, the total power constraint can be written as

$$
\sum_{i=1}^{N} P_{i}+a \sum_{i=1}^{N} P_{i}=P_{t}
$$

where $P_{t}$ is the fixed power budget of the network.

On the other hand, by considering the worst case, (24) can be bounded as

$$
\begin{aligned}
\overline{\operatorname{PEP}_{F}} \leq & \frac{\left(\left(1 /\left(1+\bar{g} \sum_{k=1}^{N} P_{k} d_{\min }^{2} / 8 \sigma^{2}\right)\right)\left(1 /\left(1+a r_{r} \sum_{k=1}^{N} P_{k} d_{\min }^{2} / 8 \sigma^{2}\right)\right)+1 /\left(1+\bar{r} \sum_{k=1}^{N} P_{k} d_{\min }^{2} / 8 \sigma^{2}\right)\right)}{12} \\
& +\frac{\left(1 /\left(1+\bar{g} \sum_{k=1}^{N} P_{k} d_{\min }^{2} / 6 \sigma^{2}\right)\left(1 /\left(1+a r_{r} \sum_{k=1}^{N} P_{k} d_{\min }^{2} / 6 \sigma^{2}\right)\right)+1 /\left(1+\bar{r} \sum_{k=1}^{N} P_{k} d_{\min }^{2} / 6 \sigma^{2}\right)\right)}{4},
\end{aligned}
$$


TABLE 1: Comparisons of the optimal power coefficients.

\begin{tabular}{lc}
\hline Work & Closed-form expression of $a$ \\
\hline 15$]$ & $a=\frac{P_{t}}{P_{t}+\left(\left(8 \sigma^{2} / d_{\min }^{2}\right) 2 \bar{r}-\sqrt{4 \bar{g} r_{r} \bar{r} P_{t}\left(8 \sigma^{2} / d_{\min }^{2}\right)+4 \bar{g}^{2}\left(8 \sigma^{2} / d_{\text {min }}^{2}\right)^{2}}\right) / 2 \bar{g} r_{r}}-1$ \\
Proposed & $a=\frac{\sqrt{\bar{r}^{2} \sigma^{4}+C \bar{g} r_{r} \bar{r}\left(\sigma / d_{\min }\right)^{2} P_{t}}-\bar{r} \sigma^{2}}{C \sum_{k=1}^{N} P_{k} \bar{g} r_{r}}$
\end{tabular}

where $\bar{g}=(1 / N) \sum_{k=1}^{N} g_{k}, \bar{r}=(1 / N) \sum_{k=1}^{N} r_{k} \cdot d_{\min }$ is the minimum distance of the CFNC constellation, which can be expressed as

$$
d_{\min }=\min _{i \neq j}\left|\left[e^{j \varphi_{1}}, \ldots, e^{j \varphi_{N}}\right]^{T} \cdot\left[\mathbf{X}_{i}-\mathbf{X}_{j}\right]\right|
$$

By substituting (25) into (26), $\overline{\mathrm{PEP}_{F}}$ can be simply expressed as

$$
\begin{aligned}
& \overline{\operatorname{PEP}_{F}} \\
& \leq \frac{\left(\left(1 /\left(1+\bar{g}\left(P_{t}-a \sum_{k=1}^{N} P_{k}\right) d_{\text {min }}^{2} / 8 \sigma^{2}\right)\right)\left(1 /\left(1+a r_{r} \sum_{k=1}^{N} P_{k} d_{\text {min }}^{2} / 8 \sigma^{2}\right)\right)+1 /\left(1+\bar{r}\left(P_{t}-a \sum_{k=1}^{N} P_{k}\right) d_{\text {min }}^{2} / 8 \sigma^{2}\right)\right)}{12} \\
& \quad+\frac{\left(\left(1 /\left(1+\bar{g}\left(P_{t}-a \sum_{k=1}^{N} P_{k}\right) d_{\min }^{2} / 6 \sigma^{2}\right)\right)\left(1 /\left(1+a r_{r} \sum_{k=1}^{N} P_{k} d_{\min }^{2} / 6 \sigma^{2}\right)\right)+1 /\left(1+\bar{r}\left(P_{t}-a \sum_{k=1}^{N} P_{k}\right) d_{\min }^{2} / 6 \sigma^{2}\right)\right)}{4} .
\end{aligned}
$$

Using the approximation $1 /(1+x) \rightarrow 1 / x$ when $x$ is in high SNR region, (28) can be simplified as

$$
\begin{aligned}
\overline{\mathrm{PEP}_{F} \leq} & \frac{\left(64 \sigma^{4} / \bar{g} r_{r} a\left(P_{t}-a \sum_{k=1}^{N} P_{k}\right) \sum_{k=1}^{N} P_{k} d_{\text {min }}^{4}+8 \sigma^{2} / \bar{r}\left(P_{t}-a \sum_{k=1}^{N} P_{k}\right) d_{\text {min }}^{2}\right)}{12} \\
& +\frac{\left(36 \sigma^{4} / \bar{g} r_{r} a\left(P_{t}-a \sum_{k=1}^{N} P_{k}\right) \sum_{k=1}^{N} P_{k} d_{\min }^{4}+6 \sigma^{2} / \bar{r}\left(P_{t}-a \sum_{k=1}^{N} P_{k}\right) d_{\text {min }}^{2}\right)}{4} \\
\leq & \frac{43}{3} \frac{\sigma^{4}}{\bar{g} r_{r} a\left(P_{t}-a \sum_{k=1}^{N} P_{k}\right) \sum_{k=1}^{N} P_{k} d_{\text {min }}^{4}}+\frac{13}{6} \frac{\sigma^{2}}{\bar{r}\left(P_{t}-a \sum_{k=1}^{N} P_{k}\right) d_{\text {min }}^{2}} .
\end{aligned}
$$

We employ (29) as the closed expression of $\overline{\mathrm{PEP}_{F}}$, and the optimization of the power coefficient $a$ can be obtained by solving the following equation as

$$
\begin{aligned}
\frac{\partial \overline{\mathrm{PEP}_{F}}}{\partial a}= & -\frac{43}{3} \frac{\sigma^{4}\left(P_{t}-2 a \sum_{k=1}^{N} P_{k}\right)}{\bar{g} r_{r} a^{2}\left(P_{t}-a \sum_{k=1}^{N} P_{k}\right)^{2} \sum_{k=1}^{N} P_{k} d_{\text {min }}^{4}} \\
& +\frac{13}{6} \frac{\sigma^{2} \sum_{k=1}^{N} P_{k}}{\bar{r}\left(P_{t}-a \sum_{k=1}^{N} P_{k}\right)^{2} d_{\min }^{2}}=0 .
\end{aligned}
$$

Multiplying both sides of (30) by $a^{2}\left(P_{t}-a \sum_{k=1}^{N} P_{k}\right)^{2}$ leads to

$$
\frac{86 \sigma^{4}\left(P_{t}-2 a \sum_{k=1}^{N} P_{k}\right)}{\bar{g} r_{r} d_{\min }^{4}}-\frac{13\left(\sigma a \sum_{k=1}^{N} P_{k}\right)^{2}}{\bar{r} d_{\min }^{2}}=0 .
$$

We denote $C=13 / 86$ and $\sum_{k=1}^{N} P_{k}=P_{t 1}$; thus (31) can be simplified as

$$
C P_{t 1}^{2} \bar{g} r_{r} a^{2}+2 \bar{r} P_{t 1} a \sigma^{2}-\bar{r}\left(\frac{r}{d_{\min }}\right)^{2} P_{t}=0 .
$$


The roots of (32) with respect to $a$ can be expressed as

$$
a=\frac{ \pm \sqrt{\bar{r}^{2} \sigma^{4}+C \bar{g} r_{r} \bar{r}\left(\sigma / d_{\text {min }}\right)^{2} P_{t}}-\bar{r} \sigma^{2}}{C P_{t 1} \bar{g} r_{r}} .
$$

By abandoning the negative root, the optimal value of $a$ is obtained. Table 1 compares this result with the one in [15], and it shows that our expression is more straightforward to reveal the relationship between $a$ and the topology parameters.

Therefore, we utilize the obtained expression of $a$ to make the insightful analysis of the topology parameters and determine the proper power allocation. The results are presented as follows.
(1) When $\sigma^{2} / d_{\min }^{2} \rightarrow 0$, the proposed expression tends to

$$
a=\frac{\sqrt{\bar{r}^{2} \sigma^{4}}-\bar{r} \sigma^{2}}{C \sum_{k=1}^{N} P_{k} \bar{g} r_{r}}=0
$$

Equation (34) reveals that when the network works in the high SNR region, the optimal policy is to allocate the power budget to all the sensor nodes without employing the relay node, which helps to avoid error detection.

(2) The derivative of the proposed expression with respect to $\bar{g}$ can be obtained as

$$
\frac{\partial a}{\partial \bar{g}}=\frac{C r_{r} \bar{r}\left(\sigma / d_{\text {min }}\right)^{2} P_{t} \bar{g} / 2 \sqrt{\bar{r}^{2} \sigma^{4}+C \bar{g} r_{r} \bar{r}\left(\sigma / d_{\text {min }}\right)^{2} P_{t}}-\left(\sqrt{\bar{r}^{2} \sigma^{4}+C \bar{g} r_{r} \bar{r}\left(\sigma / d_{\text {min }}\right)^{2} P_{t}}-\bar{r} \sigma^{2}\right)}{C \sum_{k=1}^{N} P_{k} \bar{g}^{2} r_{r}} .
$$

By using relationship $\sqrt{x^{2}+b}-x=b /\left(\sqrt{x^{2}+b}+x\right)$, there are

$$
\sqrt{\bar{r}^{2} \sigma^{4}+C \bar{g} r_{r} \bar{r}\left(\frac{\sigma}{d_{\min }}\right)^{2} P_{t}}-\bar{r} \sigma^{2}
$$

$$
=\frac{C \bar{g} r_{r} \bar{r}\left(\sigma / d_{\min }\right)^{2} P_{t}}{\sqrt{\bar{r}^{2} \sigma^{4}+C \bar{g} r_{r} \bar{r}\left(\sigma / d_{\min }\right)^{2} P_{t}}+\bar{r} \sigma^{2}} .
$$

Substituting (38) into (37), it leads to

$$
\frac{\partial a}{\partial \bar{g}}=\frac{\bar{r}\left(\sigma / d_{\min }\right)^{2} P_{t}\left(1 / 2 \sqrt{\bar{r}^{2} \sigma^{4}+C \bar{g} r_{r} \bar{r}\left(\sigma / d_{\min }\right)^{2} P_{t}}-1 /\left(\sqrt{\bar{r}^{2} \sigma^{4}+C \bar{g} r_{r} \bar{r}\left(\sigma / d_{\min }\right)^{2} P_{t}}+\bar{r} \sigma^{2}\right)\right)}{\sum_{k=1}^{N} P_{k} \bar{g}}<0 .
$$

Equation (37) demonstrates that $a$ is the monotone decreasing function given that $\bar{g}$ grows. This result indicates that when the sensor nodes locate close to FC, more power should be allocated to them, because the direct links between the sensor nodes and FC are in good quality. On the contrary, when the sensor nodes locate far from FC, the quality of the direct links are poor, so more power should be allocated to the cooperative relay for reliable transmission.

(3) The derivative of the obtained expression with respect to $r_{r}$ can be similarly obtained as

$$
\frac{\partial a}{\partial r_{r}}=\frac{\bar{r}\left(\sigma / d_{\text {min }}\right)^{2} P_{t}\left(1 / 2 \sqrt{\bar{r}^{2} \sigma^{4}+C \bar{g} r_{r} \bar{r}\left(\sigma / d_{\text {min }}\right)^{2} P_{t}}-1 /\left(\sqrt{\bar{r}^{2} \sigma^{4}+C \bar{g} r_{r} \bar{r}\left(\sigma / d_{\text {min }}\right)^{2} P_{t}}+\bar{r} \sigma^{2}\right)\right)}{\sum_{k=1}^{N} P_{k} r_{r}}<0 .
$$

The result shows that $a$ is the monotone decreasing function when $r_{r}$ grows, and it reveals that when the relay locates close to FC, more power should be allocated to the sensor nodes. This insight is reasonable, because in that scenario the link between the relay and FC is in good quality, so the end-to-end transmission error rate is dominated by the error detection at relay. If more power is allocated to the sensor nodes, the received SNR at relay can be improved, which is helpful to mitigate error propagation.

(4) The insightful relationship between $a$ and $\bar{r}$ is obtained as

$$
\frac{\partial a}{\partial \bar{r}}=\frac{\left(2 \bar{r} \sigma^{4}+C \bar{g} r_{r}\left(\sigma^{2} / d_{\min }^{2}\right) P_{t}\right) / 2 \sqrt{\bar{r}^{2} \sigma^{4}+C \bar{g} r_{r} \bar{r}\left(\sigma / d_{\min }\right)^{2} P_{t}}-\sigma^{2}}{C \sum_{k=1}^{N} P_{k} \bar{g} r_{r}} .
$$


We denote $w=C \bar{g} r_{r} P_{t} / \bar{r} \sigma^{2} d_{\text {min }}^{2}$, and (39) can be simplified as

$$
\frac{\partial a}{\partial \bar{r}}=\frac{C \bar{g} r_{r} P_{t}^{2} / \bar{r}^{2} \sigma^{2} d_{\min }^{4}}{4 \sum_{k=1}^{N} P_{k} \sqrt{1+w}(1+0.5 w+\sqrt{1+w})}>0 .
$$

The above result shows that $a$ is the monotone increasing function with respect to $\bar{r}$. In fact, when the relay node locates close to the sensor nodes, error detection is slice, so the optimal policy is to fully utilize the cooperative relay by allocating more power to it.

\section{Simulation Results}

In this section, we examine the detection performance of the obtained power coefficient by employing the one of [15] as the benchmark. Moreover, the theoretical analysis on the topology parameters presented in Section 3 is also evaluated. MATLAB is employed to build simulation platform. The probability of error and receiver operating characteristics (ROC) curves are simulated as the performance metrics through the Monte-Carlo method. In the simulation of probability of error, it is assumed that $P\left(H_{0}\right)=P\left(H_{1}\right)=0.5$ in (13), while, in the simulation of ROC curves, $P\left(H_{0}\right)$ and $P\left(H_{1}\right)$ are changed for each simulated point. Simulation length is set as 100 million times per point. Each sensor node $S_{i}$ has identical false alarm and detection probabilities as $P_{D k}=0.5$ and $P_{F k}=0.05$. Also, we assume that the power budget $P_{t}=2$ per time slot. In the simulations, the signal-to-noise (SNR) is defined as

$$
\mathrm{SNR}(\text { in } \mathrm{dB})=10 \log _{10}\left(\frac{P_{t} T_{0}}{2 \sigma^{2}}\right),
$$

in which $T_{0}=0.5$ is the time slot duration. Since $P_{t}$ and $T_{0}$ are fixed, we supply different SNRs by changing $\sigma^{2}$ in the simulation. The network topologies are modeled by the path loss coefficients, through which both the symmetric and asymmetric scenarios are considered. Here, we assume the distance between $S_{1}$ and FC is 1 , and $g_{1}=1$ is as the reference distance. The other path loss coefficients are given as gains or loss relative to it, that is, $r$ (in $\mathrm{dB})=10 \log _{10}\left(r / g_{1}\right)$.

Figure 3 shows the probabilities of error for the two compared schemes. The metric is simulated under a symmetric topology with $g_{1}=g_{2}=0 \mathrm{~dB}, r_{1}=r_{2}=10.45 \mathrm{~dB}$, $r_{r}=3.10 \mathrm{~dB}$, and $N=2$, in which the distance from the relay node to each sensor node is the same, as well as the one from FC to each sensor node. It can be observed that as SNR grows, the gap between the two schemes reduces; however, the proposed scheme is always superior to the benchmark in the same SNR region. This result verifies the effectiveness of the proposed optimization in Section 3 and shows that the accuracy improvement of approximation for $Q$ function is able to yield better transmission reliability.

Figure 4 shows the ROC curves of the two compared schemes in the same topology with different SNR values. It can be seen that the proposed scheme outperforms the benchmark in each SNR. In the other words, the proposed scheme has higher probability of detection than the benchmark under

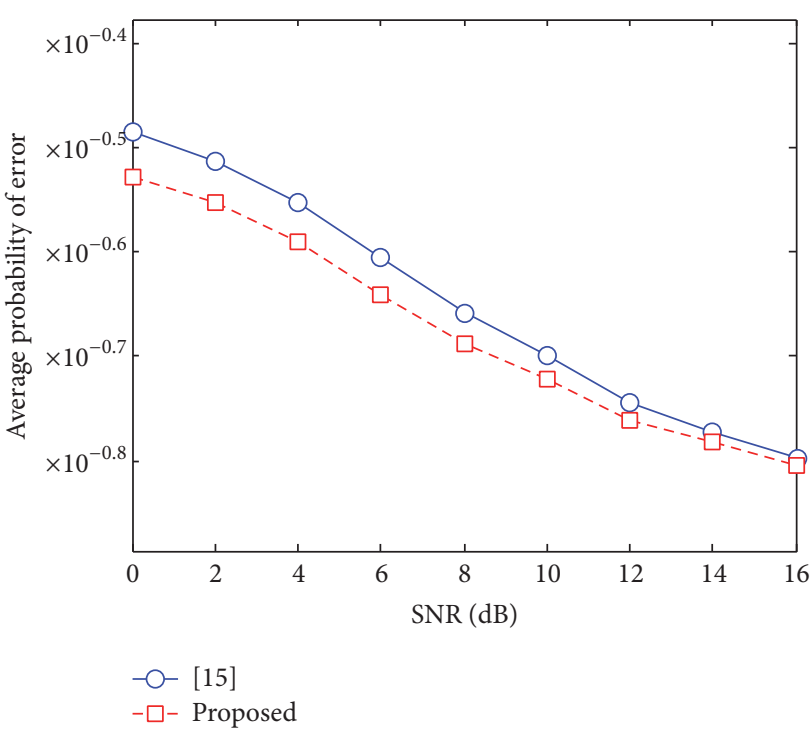

FIGURE 3: Probability of error comparisons for $N=2, g_{1}=g_{2}=$ $0 \mathrm{~dB}, r_{1}=r_{2}=10.45 \mathrm{~dB}, r_{r}=3.10 \mathrm{~dB}$.

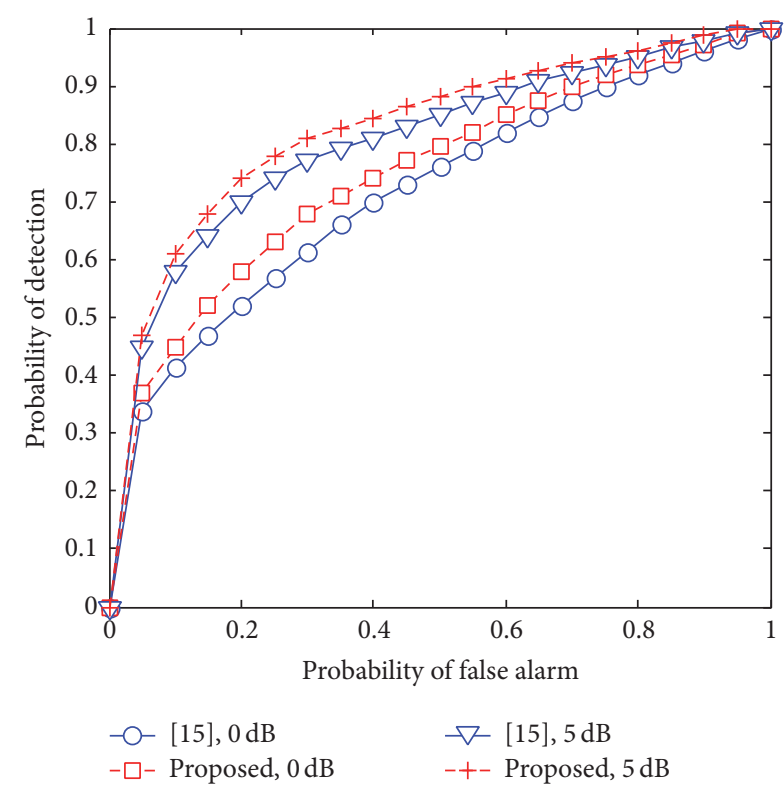

FIGURE 4: ROC curve comparison for $N=2, g_{1}=g_{2}=0 \mathrm{~dB}, r_{1}=$ $r_{2}=10.45 \mathrm{~dB}, r_{r}=3.10 \mathrm{~dB}$.

the same probability of false alarm. The results demonstrate that the proposed power allocation is helpful to improve the detection performance of the network.

Besides symmetric topology, the asymmetric topology is also employed to evaluate the scheme. Figure 5 depicts the probability of error when the relay node is closer to $S_{1}$, and the distance from $S_{2}$ to FC varies. It can be seen that although both compared schemes suffer from the decrease of $g_{2}$, the proposed scheme is superior to the benchmark. In fact, the relay node locates farer from $S_{2}$, so the error detection at the relay is dominated by the link between them. Meanwhile, when $S_{2}$ locates farther from FC, the $S_{2}$ to-FC link also demonstrates the detection of FC. Hence, 


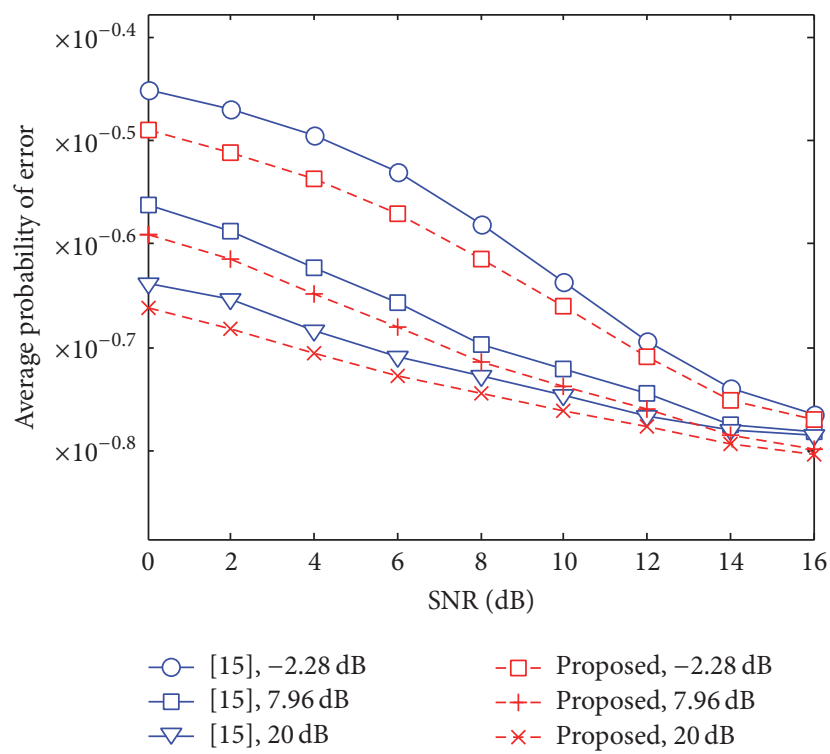

FIGURE 5: Probability of error comparison under various $g_{2}$ for $N=$ $2, g_{1}=0 \mathrm{~dB}, r_{1}=10.45 \mathrm{~dB}, r_{2}=r_{r}=3.10 \mathrm{~dB}$.

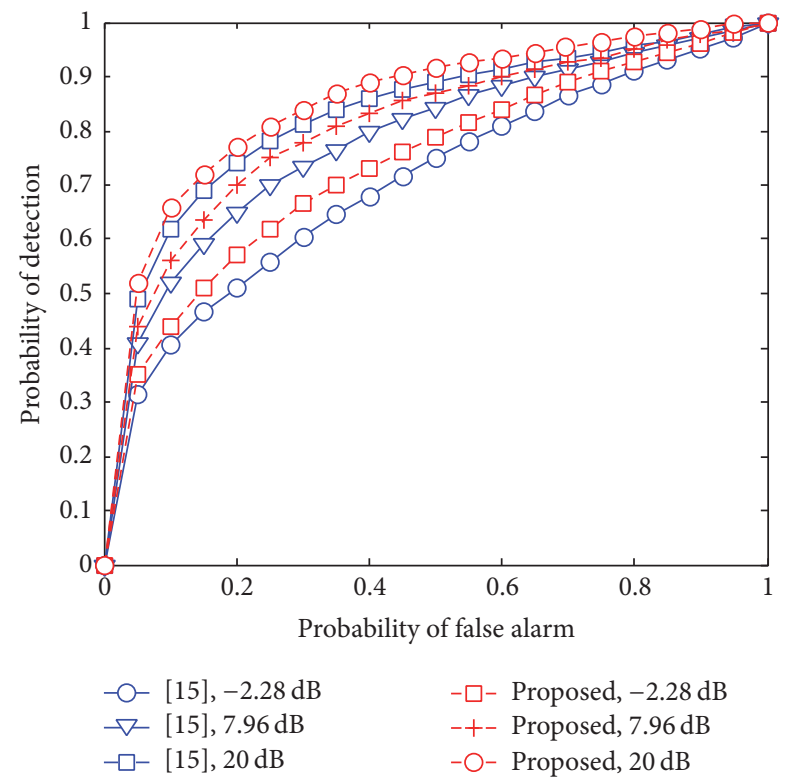

FIGURE 6: ROC curve comparison under various $g_{2}$ for $N=2, g_{1}=$ $0 \mathrm{~dB}, r_{1}=10.45 \mathrm{~dB}, r_{2}=r_{r}=3.10 \mathrm{~dB}$, and $\mathrm{SNR}=0 \mathrm{~dB}$.

the variety of $g_{2}$ influences the performance of the network largely. However, since the proposed scheme can offer more accurate power allocation to deal with the topology variety, it yields better performance. In the figure, the performance gap is more obvious when $S_{2}$ locates far from FC, which also verifies the accuracy improvement of the power allocation by the proposed optimization. Similarly, Figure 6 shows the corresponding curves of the asymmetric topology when $\mathrm{SNR}=0 \mathrm{~dB}$. The result also shows that the proposed scheme outperforms the benchmark.

We also evaluate the proposed scheme in a more complex scenario in Figure 7, in which $N=4$ and the distance

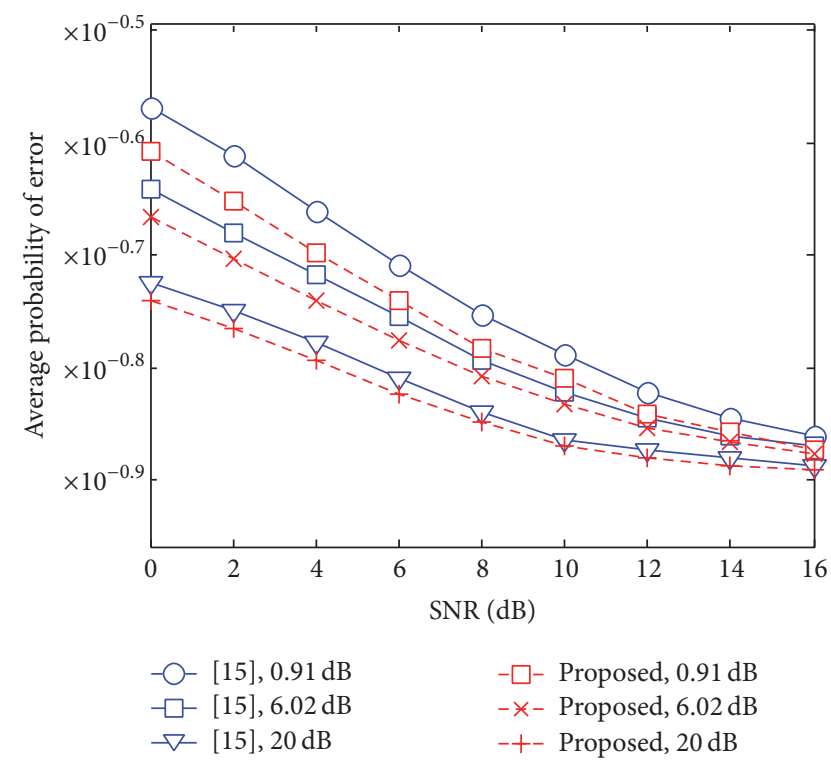

FIgURE 7: Probability of error comparison under various $g_{4}$ for $N=$ $4, g_{1}=0 \mathrm{~dB}, g_{2}=g_{3}=r_{r}=0.91 \mathrm{~dB}, r_{1}=r_{2}=r_{3}=r_{4}=13.98 \mathrm{~dB}$.

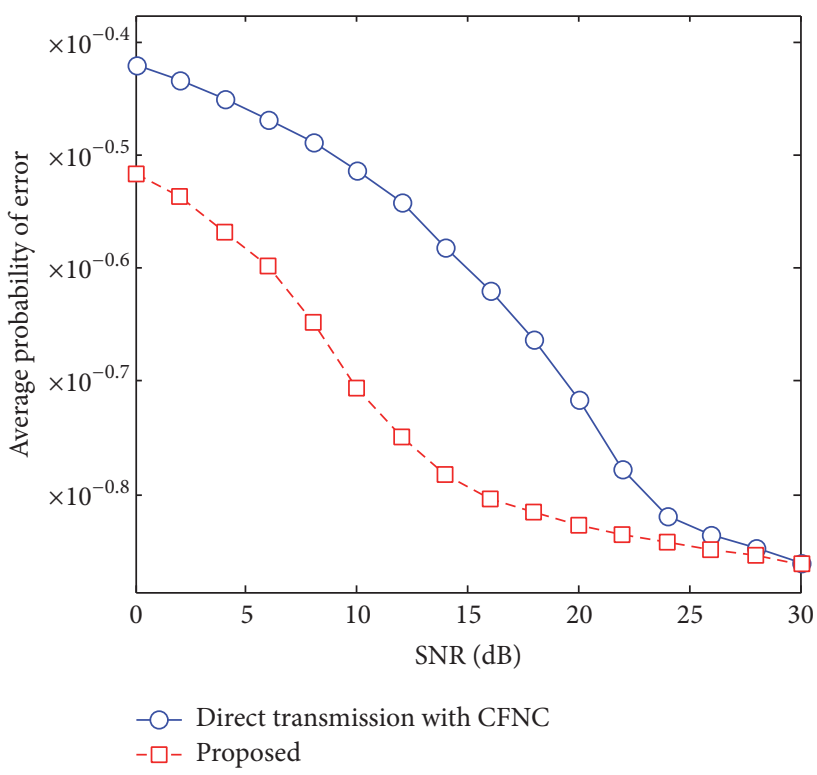

FIGURE 8: The impact of the high SNR on the probability of error with symmetric topology and $N=2$.

from each sensor node to the relay node is identical. Similar to the situation in Figure 5, the decrease of $g_{4}$ also deteriorates the performance of both schemes. Consistently, the proposed scheme has a better transmission reliability than the benchmark for each $g_{4}$. To conclude, the above results show that the proposed optimization is constructive to improve the accuracy of power allocation, as well as the detection performance of the considered WSN.

Next, we examine the theoretical analysis presented in Section 3. Figure 8 depicts the impact of the high SNR on the proposed scheme. The employed simulation parameters are the same as the ones in Figure 3. Here, we use direct 


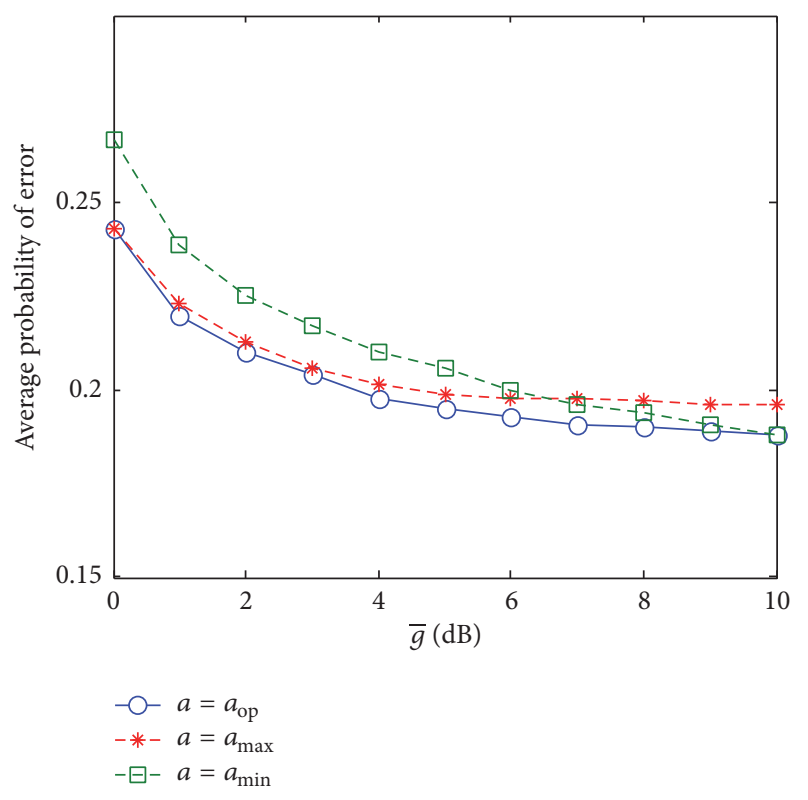

FIGURE 9: The impact of parameter $\bar{g}$ on the probability of error with symmetric topology and $N=2, \mathrm{SNR}=5 \mathrm{~dB}$.

transmission with CFNC as the benchmark, in which the power budget is equally allocated to the sensor node without considering the relay node. The figure shows that, in low and medium SNR regions, the proposed scheme outperforms direct transmission with considerable gap. However, in high SNR region, the gap is negligible. This result demonstrates that, in the high SNR region, the optimal power allocation is close to direct transmission, which corresponds well with the analysis in Section 3. So the simulation and analysis provide an insight that, in high SNR region, direct transmission is a simple yet efficient policy comparing with power allocation.

Figures 9, 10, and 11 show the impacts of parameters $\bar{g}, r_{r}$, and $\bar{r}$ on the proposed scheme, respectively. In these simulations, $\mathrm{SNR}=5 \mathrm{~dB}$, and the other parameters are the same as the one in Figure 3. In Figure 9, three power coefficients are compared, in which $a_{\mathrm{op}}$ is calculated according to the proposed expression for in Table 1 for each simulated value of $\bar{g}$, while $a_{\max }$ and $a_{\min }$ are by the expression when $\bar{g}=0$ and $10 \mathrm{~dB}$, respectively. In other words, $a_{\mathrm{op}}$ varies for each $\bar{g}$ and falls into region $\left[a_{\min }, a_{\max }\right]$. It can be observed that, compared with the two fixed values, $a_{\mathrm{op}}$ obtains better transmission reliability. This result shows that when the sensor nodes move towards FC, the proper transmission policy is to allocate more power to the sensor nodes.

Figure 10 also demonstrates that $a_{\mathrm{op}}$ is superior to $a_{\max }$ and $a_{\min }$. This result reveals that power adjustment is constructive for the transmission performance when the relay moves towards FC. Besides, it shows that, in this case, more power should be allocated to the sensor nodes. Figure 11 depicts the comparisons when the relay node moves towards the sensor nodes. Similarly, $a_{\mathrm{op}}$ yields optimal performance, while it also shows that more power should be allocated to the relay in this case. The above results verify the corresponding analysis in Section 3 and show that the proposed expression

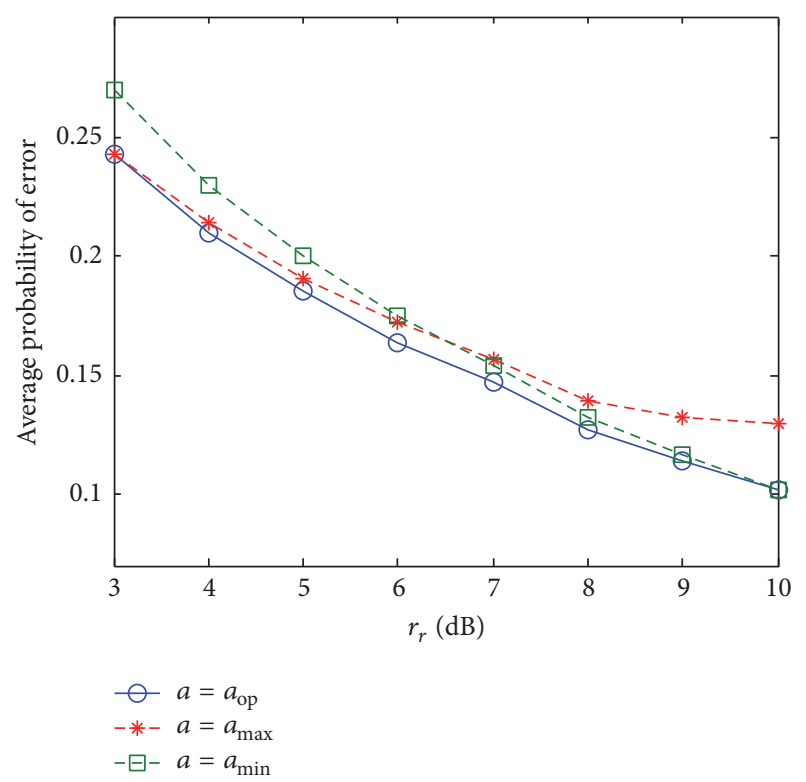

FIgURE 10: The impact of parameter $r_{r}$ on the probability of error with symmetric topology and $N=2, \mathrm{SNR}=5 \mathrm{~dB}$.

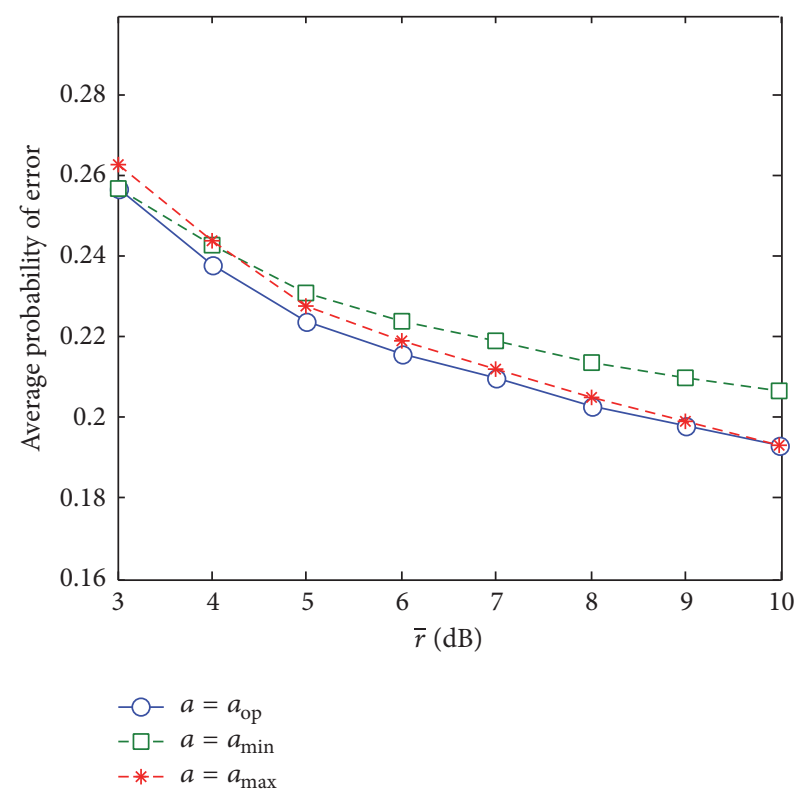

FIGURE 11: The impact of parameter $\bar{r}$ on the probability of error with symmetric topology and $N=2, \mathrm{SNR}=5 \mathrm{~dB}$.

of $a$ is effective in analyzing the impacts of the topology parameters and providing proper power allocation for each scenario.

\section{Conclusion}

This paper has investigated the power allocation for the CFNC-based DD in cooperative relay assisted WSN. In order to improve the accuracy of optimization, the closed expression of the PEP bound for the considered network is derived by utilizing a high accuracy approximation of $Q$ 
function. According to the obtained expression, the power allocation coefficient of the relay is optimized, while some useful insights on the relationship between the power allocation and topology parameters are also provided. Simulations demonstrate that the proposed optimization outperforms the benchmark [15] in terms of detection performance. Moreover, the impacts of the topology parameters on the power allocation are also verified. Therefore, the obtained expression of the power coefficient and the insights are helpful for the power allocation design in the considered WSN.

\section{Competing Interests}

The authors declare that they have no competing interests.

\section{Acknowledgments}

This work was supported by the National Basic Research Program of China (973 Program) (Grant no. 2013CB329104), the National High Technology Research and Development Program of China (863 program) (Grant no. 2014AA01A705), and the National Natural Science Foundations of China (Grants no. 61601238, no. 61372124, and no. 61427801).

\section{References}

[1] I. F. Akyildiz, W. Su, Y. Sankarasubramaniam, and E. Cayirci, "A survey on sensor networks," IEEE Communications Magazine, vol. 40, no. 8, pp. 102-105, 2002.

[2] C.-Y. Chong and S. P. Kumar, "Sensor networks: evolution, opportunities, and challenges," Proceedings of the IEEE, vol. 91, no. 8, pp. 1247-1256, 2003.

[3] P. K. Varsheny, Distributed Detection and Data Fusion, Springer, New York, NY, USA, 1996.

[4] I. Y. Hoballah and P. K. Varshney, "Distributed Bayesian signal detection," IEEE Transactions on Information Theory, vol. 35, no. 5, pp. 995-1000, 1989.

[5] S. C. A. Thomopoulos, R. Viswanathan, and D. K. Bougoulias, "Optimal distributed decision fusion," IEEE Transactions on Aerospace and Electronic Systems, vol. 25, no. 5, pp. 761-765, 1989.

[6] M. Kam, Q. Zhu, and W. S. Gray, "Optimal data fusion of correlated local decisions in multiple sensor detection systems," IEEE Transactions on Aerospace and Electronic Systems, vol. 28, no. 3, pp. 916-920, 1992.

[7] P. Willett, P. F. Swaszek, and R. S. Blum, “The good, bad, and ugly: distributed detection of a known signal in dependent gaussian noise," IEEE Transactions on Signal Processing, vol. 48, no. 12 , pp. 3266-3279, 2000.

[8] S. C. A. Thomopoulos and L. Zhang, "Distributed decision fusion in the presence of networking delays and channel errors," Information Sciences, vol. 66, no. 1-2, pp. 91-118, 1992.

[9] B. Chen, R. Jiang, T. Kasetkasem, and P. K. Varshney, "Channel aware decision fusion in wireless sensor networks," IEEE Transactions on Signal Processing, vol. 52, no. 12, pp. 3454-3458, 2004.

[10] R. Niu, B. Chen, and P. K. Varshney, "Fusion of decisions transmitted over Rayleigh fading channels in wireless sensor networks," IEEE Transactions on Signal Processing, vol. 54, no. 3, pp. 1018-1027, 2006.
[11] C. R. Berger, M. Guerriero, S. Zhou, and P. Willett, "PAC vs. MAC for decentralized detection using non-coherent modulation," IEEE Transactions on Signal Processing, vol. 57, no. 9, pp. 3562-3575, 2009.

[12] D. Ciuonzo, G. Romano, and P. Salvo Rossi, "Optimality of received energy in decision fusion over Rayleigh fading diversity MAC with non-identical sensors," IEEE Transactions on Signal Processing, vol. 61, no. 1, pp. 22-27, 2013.

[13] W. Li and H. Dai, "Distributed detection in wireless sensor networks using a multiple access channel," IEEE Transactions on Signal Processing, vol. 55, no. 3, pp. 822-833, 2007.

[14] D. Ciuonzo, G. Romano, and P. S. Rossi, "Channel-aware decision fusion in distributed MIMO wireless sensor networks: decode-and-fuse vs. decode-then-fuse," IEEE Transactions on Wireless Communications, vol. 11, no. 8, pp. 2976-2985, 2012.

[15] K. Eritmen and M. Keskinoz, "Improving the performance of wireless sensor networks through optimized complex field network coding," IEEE Sensors Journal, vol. 15, no. 5, pp. 2934 2946, 2015.

[16] T. Wang and G. B. Giannakis, "Complex field network coding for multiuser cooperative communications," IEEE Journal on Selected Areas in Communications, vol. 26, no. 3, pp. 561-571, 2008.

[17] K. Eritmen and M. Keskinoz, "Distributed detection in wireless sensor networks using complex field network coding," in Proceedings of the 16th International Conference of Information Fusion (FUSION '13), pp. 989-996, IEEE, Istanbul, Turkey, July 2013.

[18] Y. Cheng and L. Yang, "An energy-efficient transmission protocol for RNC-based cooperative WSNs with partial energy harvesting nodes," International Journal of Distributed Sensor Networks, vol. 2015, Article ID 937404, 12 pages, 2015.

[19] Q. Tian and E. J. Coyle, "Optimal distributed detection in clustered wireless sensor networks," IEEE Transactions on Signal Processing, vol. 55, no. 7, pp. 3892-3904, 2007.

[20] M. Chiani, D. Dardari, and M. K. Simon, "New exponential bounds and approximations for the computation of error probability in fading channels," IEEE Transactions on Wireless Communications, vol. 2, no. 4, pp. 840-845, 2003. 

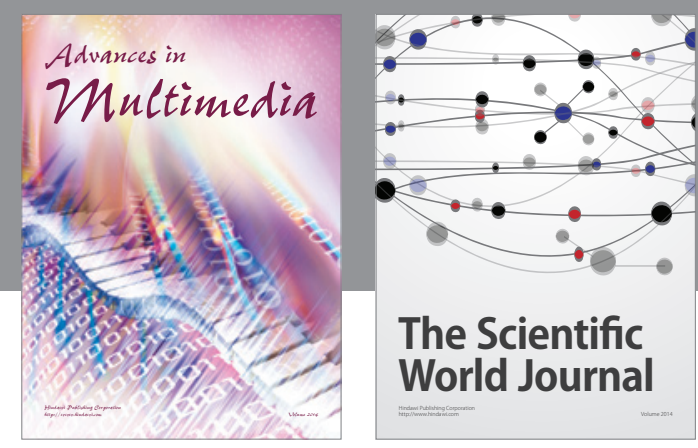

The Scientific World Journal
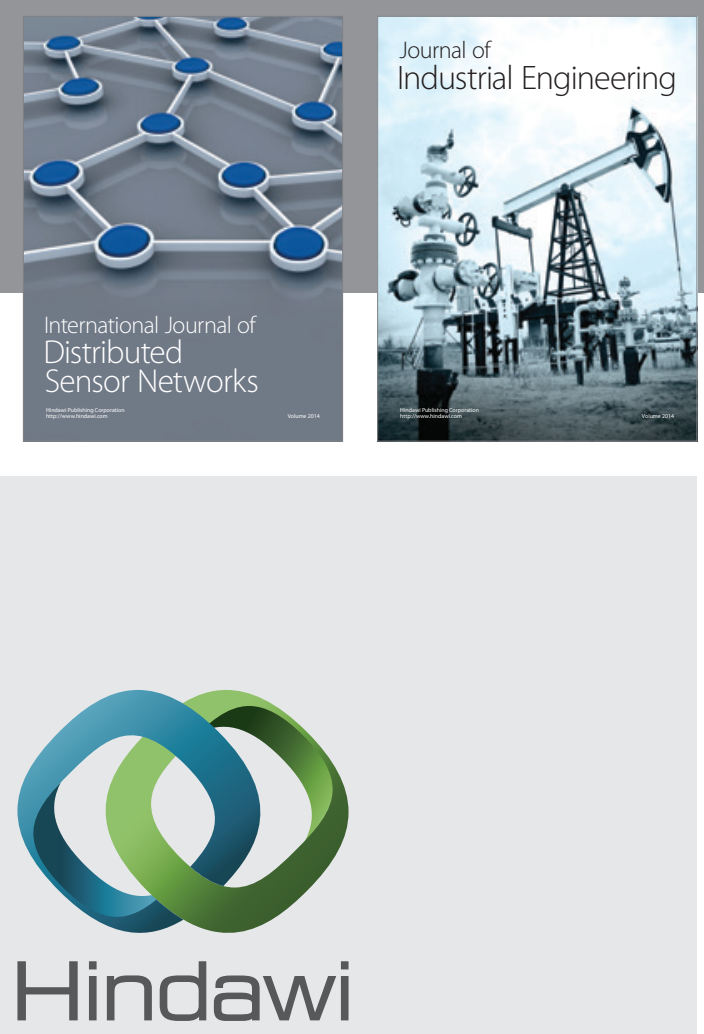

Submit your manuscripts at

http://www.hindawi.com

\section{Computer Networks} and Communications
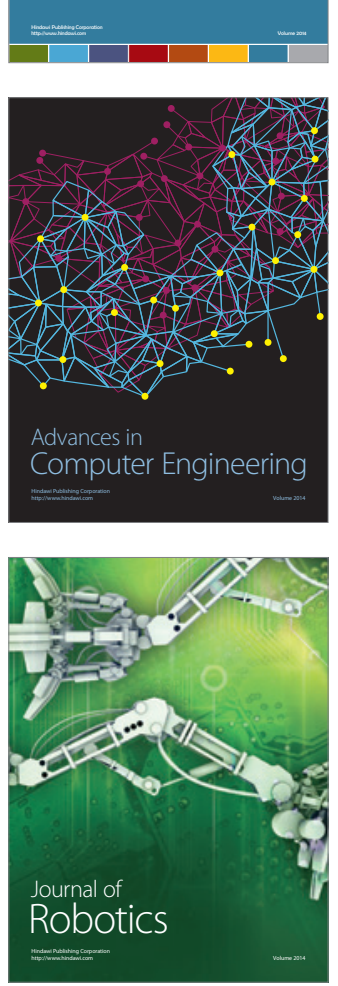
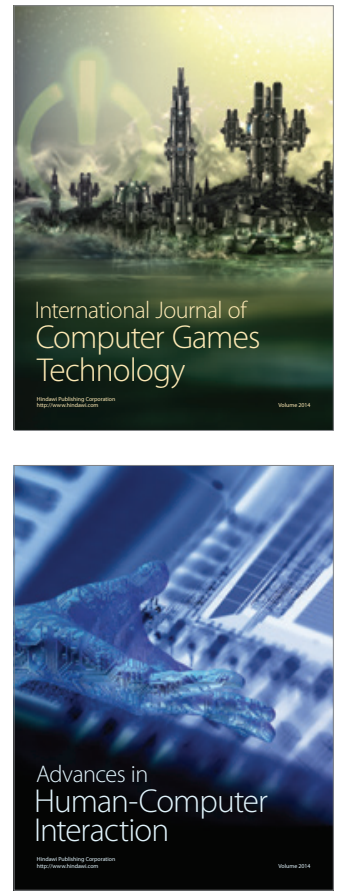
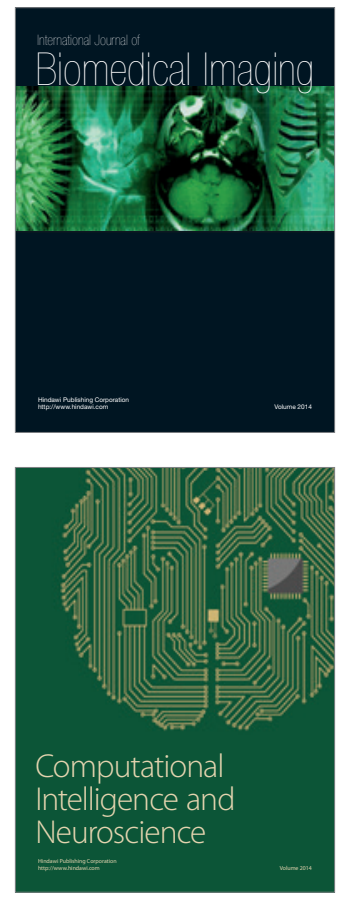
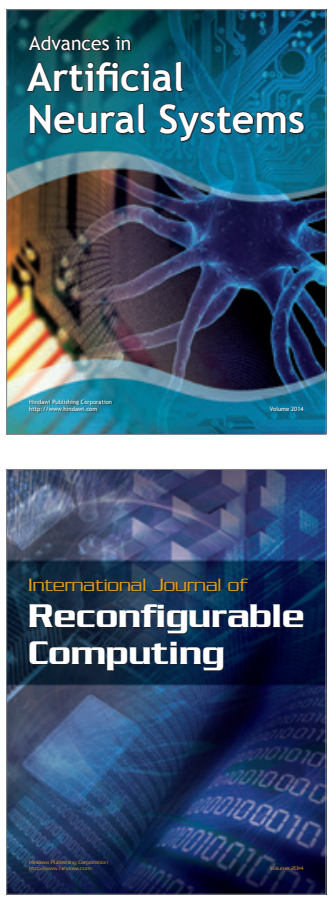
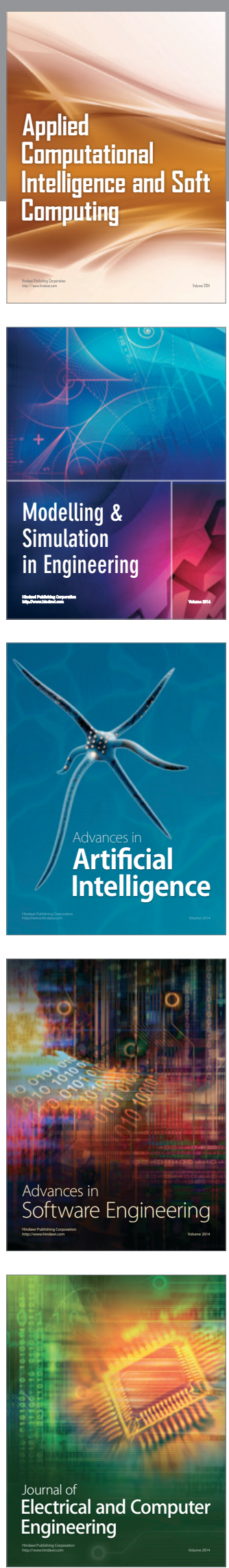